\title{
Desarrollo de la composición óptima del pan de dulce y de sal utilizando ingredientes tradicionales
}

\author{
Verónica P. Pinos $V_{.}^{1}$, Ruth Cecilia Álvarez $P^{2}$, Piercósimo Tripaldi ${ }^{3}$ \\ ${ }^{1}$ Dirección de Investigación de la Universidad de Cuenca (DIUC), Cuenca, Ecuador. \\ ${ }^{2}$ Facultad de Ciencias Químicas, Universidad de Cuenca, Cuenca, Ecuador. \\ ${ }^{3}$ Facultad de Ciencia y Tecnología, Universidad de Azuay, Cuenca, Ecuador. \\ Autor para correspondencia: veronica.pinos@ucuenca.edu.ec \\ Fecha de recepción: 16 de enero 2013 - Fecha de aceptación: 15 de mayo 2013
}

\begin{abstract}
RESUMEN
Se aplicó estadística multivariante para desarrollar dos tipos de pan, dulce y sal, respectivamente con ingredientes no-clásicos. Se consideraron varias dosificaciones de once gradientes variables, como la pepa de zambo, el maní, la quinua, el azúcar morena y la harina de plátano, esperando un nivel de aceptación mínimo de 0,97 sobre 1 puntos en ambos panes. Los límites y las condiciones de los ingredientes y los procedimientos se determinaron en base de consideraciones teóricas y resultados experimentales preliminares. En una fase inicial se aplicó el modelo factorial de Plackett-Burman que permitió la reducción de 2059 experimentos a 14, para cada tipo de pan. Cada paso del proceso fue evaluado por un panel de expertos a través encuestas de aceptación de las características organolépticas, el costo y la disposición del público a comprar. Los ingredientes relevantes fueron, respectivamente, para el pan dulce mermelada de higos y nueces, mientras que el maní para el pan de sal. En una fase posterior se optimizaron los ingredientes importantes para maximizar la composición de ambos tipos de pan. En la evaluación por parte del público el pan dulce anotó un nivel de aceptación del 90\%, mientras que el pan de sal ganó una puntuación del 78\%.
\end{abstract}

Palabras clave: Pan de dulce, pan de sal, ingredientes no clásicos, diseño experimental, optimización.

\begin{abstract}
Multivartiate statistics were applied to develop two types of bread with non-classical ingredients, respectively sweet and salty bread. Several combinations of eleven variable gradients, such as zambo seeds, peanuts, quinoa, brown sugar and banana flour, were mixed in such a way that the acceptance level of both breads was minimum 0,97 out of 1 points. The limits and condition of the ingredients, and the procedures were determined based on theoretical considerations and preliminary experimental results. In an initial phase the factorial model of Plackett-Burman was applied enabling the reduction of experiments from 2059 to 14, for each type of bread. Every step of the process was evaluated with an expert panel adopting surveys of acceptation of organoleptic characteristics, cost and the public willingness to buy. The relevant ingredients were respectively for the sweet bread mermelade of figs and nuts, while peanuts for the salt bread. In a subsequent phase the significant ingredients were optimized to maximize the composition of both bread types. In the evaluation by the public the sweet bread scored and acceptance level of $90 \%$, whereas the salt bread gained a score of $78 \%$.
\end{abstract}

Keywords: Sweet bread, salt bread, non-classical ingredients, experimental design, optimization.

\section{INTRODUCCIÓN}

El pan, los cereales, el arroz y la pasta son alimentos consumidos alrededor del mundo, la OMS los ha colocado en la base de la pirámide alimenticia (Vaclavik, 2002). De hecho, el pan constituye una base 
de alimentación desde hace 7000 u 8000 años (Mesas y Alegre, 2002). En el pan encontramos carbohidratos, donde predomina el almidón, en menor cantidad existen dextrinas, disacáridos como la maltosa y monosacáridos como la glucosa; entre las proteínas encontramos la leucina, la globulina y en mayor porcentaje gliadina y glutenina, estas últimas al unirse con agua forman el gluten; además encontramos materias grasas saturadas e insaturadas, minerales como $\mathrm{Fe}, \mathrm{Ca}$ y $\mathrm{P}$; y vitaminas generalmente del complejo B. No obstante, la mayoría de los productos disponibles en el mercado no tienen las cualidades nutricionales adecuadas. Por ello, la fortificación del alimento es una estrategia importante para una creciente población en estado de malnutrición (Güemes y col., 2009). Es de considerar que la composición nutricional del alimento reside en la calidad de los nutrientes más que en la cantidad en que se encuentran presentes. Si se considera que el pan es un producto elaborado esencialmente de harina de trigo, levadura, agua, grasa, sal y azúcar (Quaglia, 1991); la mezcla de cereales es una buena estrategia para mejorar el contenido proteico del pan y por tanto su valor alimenticio (Bourgeois y Larpent (1995) en Matos y Muñoz, 2010). Otra alternativa con este fin, es incluir alimentos con alto valor nutricional (Tejero, 1995).

La harina, componente fundamental del pan, se obtiene al moler cereales secos siendo el más empleado el trigo o Triticum Vulgare; otras harinas cereales usadas son la de maíz o Zea Mays, la de cebada o Hordeum Vulgare y la de soya o Glycine Maximus. Las cantidades de harina recomendadas considerando un cien por ciento en peso del total de harina son: trigo entre 35-100\% como único cereal que contiene cantidad suficiente de Gliadina y Glutenina para formar el gluten, maíz 50-20\%, cebada $20-35 \%$ y soya $2-10 \%$. Otros ingredientes fundamentales considerando el cien por ciento de peso en harina son: la levadura de cerveza o Saccharomyces Cervisiae, agente fermentador que con sus enzimas aprovecha los azúcares para formar alcohol y gas carbónico, se lo usa entre 2 a 4\%. Las grasas, cuya función es la de emulsionante entre las moléculas de almidón y la red glutínica, mejora el aspecto de la miga; se los usa entre 1 a 40\%. La sal y el azúcar mejoran las características organolépticas del producto, y están recomendados en dosis del 1,5 al 2,5\% y del 2 al $20 \%$ respectivamente. Otros elementos integrados al producto en busca de mejorar sus características organolépticas y nutricionales son: los frutos secos, los huevos y la leche recomendados en porcentajes del 2 al 25\%, 0 al 25\% y del 3 al $5 \%$ de cada uno. La cantidad de agua al mezclar con la harina para conseguir una consistencia estándar por regla general es de 55 a 61 partes por 100 de harina (Quaglia, 1991). Otras alternativas de ingredientes con este fin son: el almidón de achira, la harina de plátano, el maní o Arachis Hypogaea, las semillas de zambo, las semillas de girasol o Helianthus Annuus, la quinoa Chenopodium Quinoa, la alfalfa o Medicago Sativa y las frutas en general.

Los procesos básicos para la elaboración del pan son: el amasado proceso físico donde se busca la mezcla de los ingredientes para producir cambios químicos como la formación de gluten, la división y el pesado para realizar porciones, el boleado para la forma, el reposo para que la levadura leude, el formado, la fermentación, el corte y la cocción (Mesas y Alegre, 2002). Se recomienda que el amasado se realice entre 20 y $24^{\circ} \mathrm{C}$; la temperatura, humedad y el $\mathrm{pH}$ óptimo para el desarrollo de la levadura están entre 24 y $29^{\circ} \mathrm{C}, 75 \%$ de humedad y $5 \mathrm{de} \mathrm{pH}$; para el horneo se recomiendan temperaturas entre 190 a $270^{\circ} \mathrm{C}$ (Quaglia, 1991).

Si bien el elegir ingredientes, condiciones y procesos óptimos pueden asegurar la calidad del producto, es importante conocer cual será la aceptación de éste. La aceptación de un alimento depende de muchos factores, entre los que destacan sus propiedades sensoriales como su color, sabor, aroma, textura y hasta el sonido que se genera cuando se consume (Badui, 1999). Degustar un alimento significa probarlo con la intención de valorar su calidad organoléptica global en función de un modelo establecido a priori de acuerdo a la necesidad (Sancho y col., 2002). Las funciones de la degustación son las de analizar, describir, definir, juzgar y clasificar (Bourdieu, 1998).

Como se puede notar, en la tecnología de alimentos se debe considerar un sinnúmero de factores o variables para la obtención de un producto determinado, lo que puede hacer muy ardua y costosa la tarea. De hecho, en la práctica es frecuente que el investigador tenga muchas variables medidas u observadas conjuntamente que proporcionan información que muchas veces es redundante o excesiva, por cuanto la reducción de dimensiones por métodos multivariantes es una opción (Pérez, 2006). El objetivo del diseño experimental es obtener un resultado confiable para la solución de un problema con la realización de un número mínimo de experimentos (Marengo, 1994). Al hablar de experimento se considera un estudio en el que al menos una variable es manipulada y cuyas unidades son 
aleatoriamente asignadas a los distintos niveles o categorías de las variables manipuladas (Pedhazur y Pedhazur, 1991).

El diseño experimental está compuesto por: el factor o variable de interés controlada para estudiar sus efectos en una o varias respuestas; el nivel o modalidad específica dentro de un factor; el tratamiento o combinaciones de los niveles de varios factores aplicados conjuntamente a las unidades experimentales; el efecto principal o contribución de cada factor sobre la variable respuesta; y la interacción o los efectos sobre las variables respuesta bajo estudio, esto implica una relación o dependencia entre los efectos de dos o más factores. Dentro del diseño experimental se deberán considerar el ruido y la aleatorización. El ruido se compone de tres partes: el error experimental que cada unidad experimental aporta al estudio; el error de medición; y el error ambiental. La aleatorización de los tratamientos es necesaria para determinar si las variaciones se deben al error experimental o a los efectos debidos a los factores de control (Castaño y Domínguez, 2010).

Si el número de los diferentes tratamientos es muy grande con frecuencia los experimentos preliminares se realizan en cada factor en dos niveles, alto o máximo (+) y bajo o mínimo (-), por tanto para $\mathrm{k}$ factores hay $2^{\mathrm{k}}$ tratamientos diferentes, tales experimentos se llaman experimentos factoriales, de esta manera, podremos determinar qué factor tiene un efecto importante en el resultado (Navidi, 2006; Marengo, 1994). Los diseños de Plackett-Burman (1946) son diseños ortogonales que usan la matriz de Hadamard y miden la estimación insesgada de muchos efectos principales al realizarla con un número relativamente pequeño de pruebas experimentales (Box y col., 2008; Castaño y Domínguez, 2010); son de mucha utilidad cuando se puede suponer que todas las interacciones son depreciables. El diseño de Plackett-Burman coloca a cada factor en dos niveles y el número de experimentos debe ser un múltiplo de cuatro. Las variables pueden ser cualitativas o cuantitativas. El modelo toman valores de máximo (+) y mínimo (-) donde la primera línea del modelo está dada a priori y el resto del modelo se construye colocando el último número de la fila anterior al inicio de la siguiente y así sucesivamente hasta completar el modelo, la última fila está constituida por mínimos (Castaño y Domínguez, 2010; Lewis y col., 1999). Por ejemplo, la primera fila del diseño de PlackettBurman fraccionario a dos niveles, para 11 factores es: $[+,+,-,+,+,+,-,-,-,+,-]$ (Todeschini, 1998b).

Por otra parte, para optimizar los resultados encontrados, las funciones multicriterio, como las de utilidad nos ayuda a llegar a una estrategia matemática que engloba las diferentes sensaciones organolépticas y de interés en una sola respuesta que representa al experimento (Todeschini, 1998a). La optimización de la respuesta se puede analizar con el método de superficie de respuesta (Lewis y col., 1999). La metodología de superficie de respuesta (MSR) fue desarrollada por Box y Wilson en 1951 (Darghan y col., 1978). Entre los modelos más conocidos de superficie de respuesta están los que permiten la estimación de los modelos de primer y segundo orden. En esencia los modelos de superficie de respuesta son un conjunto de técnicas que se utilizan antes, durante y después del haber realizado un análisis de regresión para poder entender las características del sistema de respuesta del sistema bajo estudio (Darghan y col., 1978).

En el área de alimentos, existen diferentes estudios donde se han probado la efectividad de los diseños experimentales para la optimización de alimentos de diversa índole (Sanchez y col., 2002; Garrido y col., 2010; Salamanca y col., 2010; Saltos y Bayas, 2010; Rojas y col. 2010; Rojas y col., 2012). Y en particular, estudios demuestran que el diseño de Plackett-Burman y las funciones de utilidad son herramientas efectivas para la valoración sensorial, el control de calidad y la aceptabilidad de alimentos cárnicos, lácteos entre otros (Rojas y col., 2010; Tinoco y col., 2011). El objetivo de este estudio fue desarrollar dos tipos de pan con ingredientes saludables, uno dulce y otro de sal, a través de métodos estadísticos multivariantes para obtener un resultado total de la aceptación de los productos para un panel de expertos de al menos 0,97 sobre uno. 


\section{MATERIALES Y MÉTODOS}

\subsection{Plan de experimentación}

Para la realización de este trabajo de investigación se siguió el plan del proceso de experimentación según Marengo (1994) que considera los siguientes pasos: análisis del problema, planificación de los experimentos, ejecución de los experimentos, tratamiento estadístico de los datos y análisis de resultados. Este ciclo se repite hasta cuando se obtenga el resultado planteado. En el análisis del problema se planteó la necesidad de obtener dos panes, uno de dulce y uno de sal, con 11 ingredientes variables y un puntaje mínimo de aceptación del panel de expertos de al menos 0,97 sobre uno. Este puntaje incluía la valoración de las características organolépticas, análisis del costo e intensión de compra. En la experimentación ha aplicado cuatro etapas de experimentación, cada una de ellas acompañada por una evaluación de aceptación de los productos.

\section{Determinación del proceso}

Se eligieron las materias primas o ingredientes y sus límites por las propiedades que aportan tanto nutricionalmente como organolépticamente además de su función en la formación de masa en el proceso y en el producto terminado. Para la dosificación de las materias primas, el cálculo de los porcentajes se hizo tomando como base el peso de las harinas. En el diseño experimental, se considera a las cantidades abiertas, en este caso la unidad fue gramos. El resultado de esta etapa consistió en la determinación de la fórmula base de los dos panes.

Para la experimentación se clasificaron dos categorías de ingredientes; los que entran en la dosificación en proporción fija y los que se pueden incorporar en cantidades variables. Se consideró un total de $600 \mathrm{~g}$ de harina. Como ingredientes fijos se incluyeron a la harina de trigo $(50 \mathrm{~g})$, los huevos $(10 \mathrm{~g})$, la leche semidescremada a $35^{\circ} \mathrm{C}(20 \mathrm{~g})$, la levadura en polvo $(4 \mathrm{~g}$ para el pan de dulce y $3 \mathrm{~g}$ para el de sal), el aceite $(6 \mathrm{~g})$ y el agua $(25 \mathrm{~g}$ para el pan de dulce y $35 \mathrm{~g}$ para el de sal). La sal fue considerada fija en el pan de dulce $(0,4 \mathrm{~g})$ y el azúcar en el pan de sal $(5 \mathrm{~g})$. Como ingredientes variables y sus mínimos y máximos en gramos tanto en el pan de dulce y en el de sal se tuvo a la harina de maíz (19-9,5 y 30-15), la harina de plátano (12-6 y 10-5), la harina de soya (2-1 y 5-2,5) y a la harina de cebada (3-1,5 en ambos). Como ingredientes variables para el pan de sal estuvieron la pepa de zambo (5-2,5), el maní (3-1,5), la quinoa $(5-2,5)$ y el ajonjolí $(5-2,5)$ todos previamente tostados. Además la sal (1-0,5), brotes de alfalfa (5-2,5) y semillas de girasol (5-2,5). Como ingredientes variables en el pan de dulce tuvimos al almidón de achira (14-7), pasas (10-5), nueces (10-5), azúcar morena (25-12,5), zumo de naranja (20-10), manzana picada y pelada, tipo delicia (2010) y dulce de higo en miel de panela escurrido y troceado a $65^{\circ}$ Brix (20-10).

Además, se establecieron los procesos y las condiciones de trabajo. Todos estos parámetros fueron validados a través de una prueba piloto. El proceso inició con la recepción de materia prima, su selección y pesaje de acuerdo a dosificación. En este punto para asegurar que la elaboración del pan sea realizada siempre en condiciones constantes, se empleó una máquina para pan modelo FBM280 de la marca Franklin Chef, en su ciclo número 1 o básico de 3 horas. La incorporación de ingredientes dentro de la máquina se realizó en el siguiente orden: primero los ingredientes líquidos fijos, agua y leche a continuación el azúcar y la levadura, luego las harinas, los huevos y la sal, finalmente los ingredientes restantes, incluyendo para el pan de dulce el zumo de naranja. El ciclo de la máquina comprendió el mezclado de los ingredientes, primer amasado (10 min), primer leudado (20 min), segundo amasado (15 $\mathrm{min})$, segundo leudado (20 $\mathrm{min})$, reposo (30 $\mathrm{min})$, leudado final $(55 \mathrm{~min}) \mathrm{y}$ cocción (1h). Finalmente, el enfriamiento $(30 \mathrm{~min})$, pesaje, enfundado, etiquetado, almacenaje y control.

\section{Diseño experimental}

Se realizó para determinar cómo influía cada ingrediente variable en el producto. El modelo factorial completo para 11 variables requieren de 2059 experimentos $(211=2048+11$ al centro - valores medios establecidos para cada variable); pero con el empleo Plackett-Burman (Fig. 1) se redujeron los 
experimentos a 14 por tipo de pan, además se incluyeron dos réplicas al centro (0) para determinar el error experimental y la desviación estándar.

\begin{tabular}{llllllllllll}
\hline $\mathrm{I}$ & 1 & 2 & 3 & 4 & 5 & 6 & 7 & 8 & 9 & 10 & 11 \\
\hline 01 & + & + & - & + & + & + & - & - & - & + & - \\
02 & + & - & + & + & + & - & - & - & + & - & + \\
03 & - & + & + & + & - & - & - & + & - & + & + \\
04 & + & + & + & - & - & - & + & - & + & + & - \\
05 & + & + & - & - & - & + & - & + & + & - & + \\
06 & + & - & - & - & + & - & + & + & - & + & + \\
07 & - & - & - & + & - & + & + & - & + & + & + \\
08 & - & - & + & - & + & + & - & + & + & + & - \\
09 & - & + & - & + & + & - & + & + & + & - & - \\
10 & + & - & + & + & - & + & + & + & - & - & - \\
11 & - & + & + & - & + & + & + & - & - & - & + \\
12 & - & - & - & - & - & - & - & - & - & - & - \\
$\mathrm{RC}$ & 0 & 0 & 0 & 0 & 0 & 0 & 0 & 0 & 0 & 0 & 0 \\
$\mathrm{RC}$ & 0 & 0 & 0 & 0 & 0 & 0 & 0 & 0 & 0 & 0 & 0 \\
\hline
\end{tabular}

Figura 1. Esquema factorial de Plackett-Burman para 11 variables (columnas), 12 experimentos incluyendo 2 réplicas al centro (Consonni y Todeschini, 1998).

Donde “+” es el valor máximo, “-” es el mínimo, y el 0 representa al centro o valor medio de un determinado ingrediente en la dosificación. Las filas son los experimentos y las columnas representaron a los ingredientes variables o factores para ambos panes: 1. harina de maíz, 2. harina de plátano, 3. harina de cebada y 4. harina de soya. Para el pan de sal: 5. semillas de girasol, 6. ajonjolí, 7. pepa de zambo, 8. brotes de alfalfa, 9. quinoa, 10. maní y 11. sal. Mientras que para el pan de dulce: 5 . almidón de achira, 6. azúcar morena, 7. zumo de naranja, 8. pasas, 9. dulce de higo en panela, 10. manzanas y 11 . nueces.

\section{Optimización}

Luego de que únicamente dos ingredientes resultaran relevantes para el pan de dulce, se empleó el método de superficie de respuesta. La matriz para la experimentación fue tomada del modelo de superficie de respuesta para doce experimentos. Estos doce experimentos incluyen cuatro, donde todos los elementos (ingredientes) son constantes en su valor medio; en el resto de experimentos solo dos elementos, ingredientes, varían y el resto se mantienen como constantes. En su representación, "+" es el valor máximo, "-" es el mínimo y 0 es el punto medio o centro. Para la experimentación en concreto, la matriz mantuvo para las columnas $1,2,3,4,5,6,7,8$ y 10, de los ingredientes variables: 1. harina de maíz, 2. harina de plátano, 3. harina de cebada, 4. harina de soya, 5. almidón de achira, 6 . azúcar morena, 7. zumo de naranja, 8. pasas y 10. manzanas: $[0,0,0,0,0,0,0,0,0,0,0,0]$. Al resultar únicamente como relevantes, el dulce de higo de influencia negativa y las nueces de influencia positiva, solo estos variaron a través de los experimentos. La columna 9 correspondiente al dulce de higos en panela $[-,+,-,+,-\sqrt{2}, 0, \sqrt{ } 2,0,0,0,0,0]$ y la columna 11 a las nueces $[-,-,+,+, 0,-\sqrt{2}, 0, \sqrt{ } 2$, $0,0,0,0]$.

Para la optimización del pan de sal se realizaron 5 experimentos que incluían dos experimentos, donde todos los elementos son constantes en su valor medio. En los tres restantes experimentos solo varía un elemento. Donde, "+" representa al valor máximo, "-" el mínimo y 0 el punto medio o centro. Las columnas 1, 2, 3, 4, 5, 6, 7, 8, 9 y 11 representaron: 1. harina de maíz, 2. harina de plátano, 3. harina de cebada, 4. harina de soya, 5. semillas de girasol, 6. ajonjolí, 7. pepa de zambo, 8. brotes de alfalfa, 9. quinoa, 10. maní y 11. sal. Las columnas con excepción de la columna 10 correspondió a [0, $0,0,0,0,0,0,0,0,0,0,0]$ que indica que todos estos ingredientes se mantuvieron constantes al 
centro. Como en la fase anterior, se encontró relevante únicamente al 10. maní, de influencia negativa, solo este varió y se le asignó la columna $[-3,-2,-1,0,0]$.

Pruebas de aceptación

La calidad de las diferentes muestras de pan fue analizado por un panel de veinte personas expertas. Las que evaluaron su color, textura, sabor, olor y precio por $300 \mathrm{~g}$ de pan; la escala empleada fue de calidad (3. excelente, 2. bueno, 1. regular, 0. malo); además, se les preguntó sobre si comprarían el producto para lo que se usó una escala dicotómica (1. si, 0 no). Se normalizó las calificaciones de cada variable, esto es, color, textura, sabor, olor y precio correspondiente a cada pan, con la siguiente fórmula (Consonni y Todeschini, 1998):

$$
X_{i j}=\frac{X_{i j}-X_{j \min }}{X_{j \max }-X_{j \min }}
$$

Ec. 1

donde $X_{j \max }$ es el valor máximo de la escala y $X_{j \min }$ es el mínimo. $X_{\mathrm{ij}}$ es el valor obtenido de la encuesta. Una vez normalizados los resultados de cada experto, se determinaron las medianas del color, la textura, el sabor, el aroma y el costo. Para el deseo de compra, dicotómica, se obtuvieron modas. Los resultados fueron corregidos mediante gráficas de utilidad (ver Fig. 2). El eje de las X es el valor normalizado del puntaje obtenido en la encuesta; este se debe proyectar hasta la gráfica y luego hacia el eje Y, para lograr así el nuevo valor corregido. El puntaje total del experimento será la media de sus puntajes de sabor, color, aroma, textura, evaluación del precio e intención de adquisición. Para el sabor, adquisición y costo no existe corrección pues los valores se mantienen; para la gráfica de la textura resultan cero los valores menores a 0,5 y mayores a 0,75 mientras que los valores entre 0,5 y 0,75 puntuaron uno; para el color y aroma resultan cero los valores menores a 0,25 y mayores a 0,75 mientras que puntúan uno los valores entre 0,5 y 0,75 .

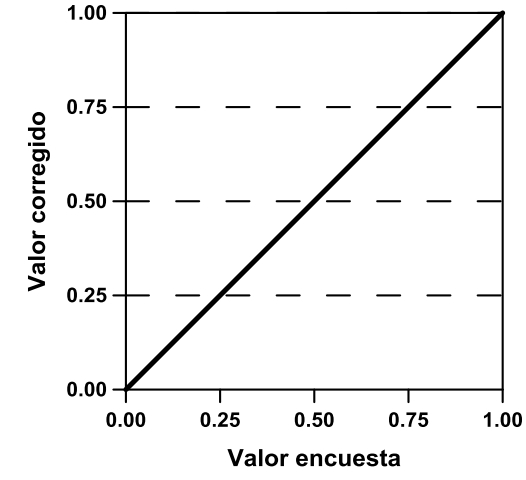

(a)

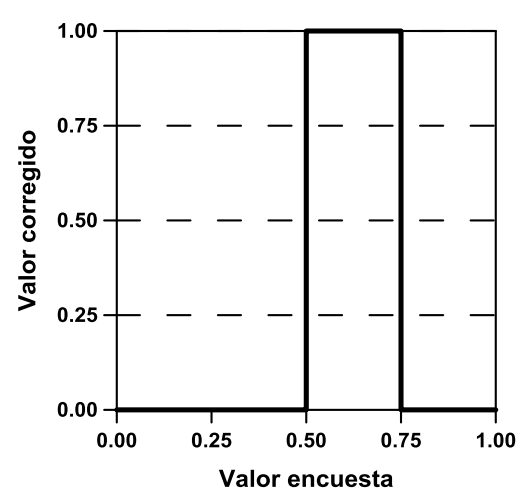

(b)

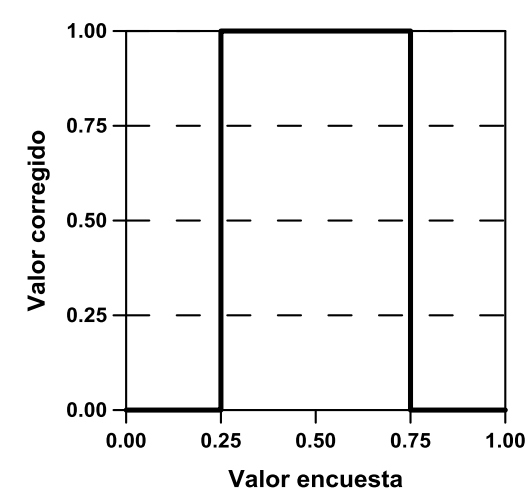

(c)

Figura 2. Gráficas de utilidad: (a) sabor, adquisición y costo; (b) textura; y (c) color y aroma.

La matriz de diseño y el vector resultados obtenidos, se procesaron aplicando la regresión multivariante de mínimos cuadrados ordinarios, donde se encontró el vector repuesta con los coeficientes de cada ingrediente, de acuerdo a la siguiente fórmula:

$$
b=\left(X^{T} X\right)^{-1} X^{T} Y
$$

donde $\mathrm{X}$ es la matriz modelo, $\mathrm{X}^{\mathrm{T}}$ su inversa, $\mathrm{Y}$ el vector respuesta $\mathrm{y}$ b es el vector columna que tiene los coeficientes del modelo.

Normal probability plot: En el vector de coeficientes, los resultados positivos son los factores de influencia positiva mientras que los negativos son los de influencia negativa. Para evidenciar la relevancia de los ingredientes, se graficó los coeficientes versus sus probabilidades (Consonni y Todeschini, 1998). Las probabilidades se encontraron con la fórmula:

$$
p_{i}=\frac{100 \%(i-0,5)}{k}
$$


donde $\mathrm{p}_{\mathrm{i}}$ es la probabilidad asociada al enésimo coeficiente, i el orden del enésimo coeficiente y $\mathrm{k}$ el número total de coeficientes calculados. La gráfica se interpreta de la siguiente manera, son relevantes los ingredientes que no pertenecen a la recta y son irrelevantes los que están contenidos en ella.

Gráfico polinomial: Se grafican las puntuaciones obtenidas por el producto versus la cantidad del ingrediente en análisis. Esta gráfica sirve para determinar la cantidad óptima del ingrediente en la formulación.

Optimización: Una vez encontradas las ecuaciones del ingrediente a optimizar, se las deriva para encontrar la cantidad óptima del ingrediente dado y lograr el puntaje máximo del producto.

Valoración: Para la valoración de los dos productos optimizados se realizaron degustaciones y encuestas de valoración de los productos, para ello, se consideró un nivel de significancia de $1 \%$, con una frecuencia del $90 \%$, se esperó una colaboración del $80 \%$. Resultando un grupo de 24 catadores de público general.

Los 24 encuestados que realizaron las pruebas de cata estuvieron entre 20 y 60 años de edad, muchos de ellos profesores y estudiantes de la Facultad de Ciencias Químicas de la Universidad de Cuenca, quienes fueron debidamente informados del objetivo y la forma de evaluar sensorialmente las distintas fórmulas de pan. Las horas y sitios se seleccionaron de manera que no interfieran en sus sentidos y tampoco reciban influencias positivas o negativas como son las horas previas y posteriores de comidas habituales. Se les realizó una encuesta ampliada a la que se usaba; es decir, además del sabor, color, aroma, textura, evaluación del precio e intención de adquisición se les preguntó acerca de la frecuencia de adquisición del producto.

\section{RESULTADOS Y DISCUSIÓN}

\subsection{Determinación de límites}

Los resultados obtenidos en la primera etapa, a través de encuestas para medir la aceptación del producto y corregidos en las gráficas de utilidad, fueron para el pan de dulce: 0,79 color y textura; 0,81 sabor; 0,75 olor; 1,00 deseo de compra con un total de 0,79 puntos; y para el pan de sal: 0,77 color; 0,71 textura; 0,72 sabor; 0,73 olor; 0,91 deseo de compra, que dan un total de 0,73 . Ambos panes tuvieron una buena aceptación, por lo que se mantuvieron los límites de ingredientes fijados, que fueron planteados desde la teoría, para el resto de la experimentación con meta de alcanzar una puntuación mínima 0,97 de su combinación.

\subsection{Diseño experimental según el modelo factorial de Plackett-Burman}

Para el pan de dulce, los parámetros de acuerdo a los valores corregidos en las gráficas de utilidad fueron: en todos los experimentos el color y aroma obtuvieron su valor máximo o 1; en la textura los experimentos 2, 3, 6, 11, 12, y las replicas al centro, $\mathrm{R}_{\mathrm{C} 1}$ y $\mathrm{R}_{\mathrm{C} 2}$ puntuaron 1 todo el resto 0; en el sabor los experimentos $\mathrm{R}_{\mathrm{C} 1}$ y $\mathrm{R}_{\mathrm{C} 2}$ obtuvieron 1 y el resto de los experimentos 0,67; en el costo los experimentos $4,5,6,10,11,12, \mathrm{R}_{\mathrm{C} 1}$ y $\mathrm{R}_{\mathrm{C} 2}$ obtuvieron 0,67 el resto 0,33 y en el deseo de compra 8 y 9 puntuaron cero y el resto 1 . De esto, los resultados totales por experimento fueron: experimento: 1 . 0,$67 ; 2$. 0,$83 ; 3$. 0,83; 4. 0,72; 5. 0,72; 6. 0,89; 7. 0,67; 8. 0,50; 9. 0,50; 10. 0,72; 11. 0,89; 12. 0,89; 13 . o $\mathrm{R}_{\mathrm{C} 1}$ (réplica al centro 1) 0,95 y 14 . o $\mathrm{R}_{\mathrm{C} 2}$ (réplica al centro 2) 0,95 . La desviación estándar y el error medido en las réplicas fueron de cero. En los resultados totales, se observa las mayores puntuaciones en los productos donde los ingredientes estaban incorporados a un nivel medio o al centro (0).

De la matriz de diseño y el vector resultados se obtuvo el vector respuesta, integrado por los siguientes coeficientes de la regresión: b1. harina de maíz $(0,28)$, b2. harina de plátano $(-0,17)$, b3 . harina de cebada $(0,17)$, b4 . harina de soya $(-0.39)$, b5. almidón de achira $(-0,28)$, b6. azúcar morena ($0,50)$, b7 zumo de naranja $(-0,06)$, b8 . pasas $(-0,50)$, b9. dulce de higos en panela $(-0,95)$, b10 manzanas $(-0,28)$ y b11 nueces $(0,83)$. Los otros ingredientes de influencia positiva fueron: la harina de maíz, la harina de cebada y las nueces mientras los ingredientes de influencia negativa fueron la harina de plátano, la harina de soya, el almidón de achira, el azúcar morena, el zumo de naranja, las pasas, el dulce de higo y las manzanas. De acuerdo a la gráfica normal probability plot (ver Fig. 3), las 
variables relevantes o que está fuera de la línea de tendencia resultaron: Las nueces (b11) de influencia positiva y el dulce de higos en panela (b9) de influencia negativa. Por lo que solo estos se consideraron para la siguiente fase, optimización.

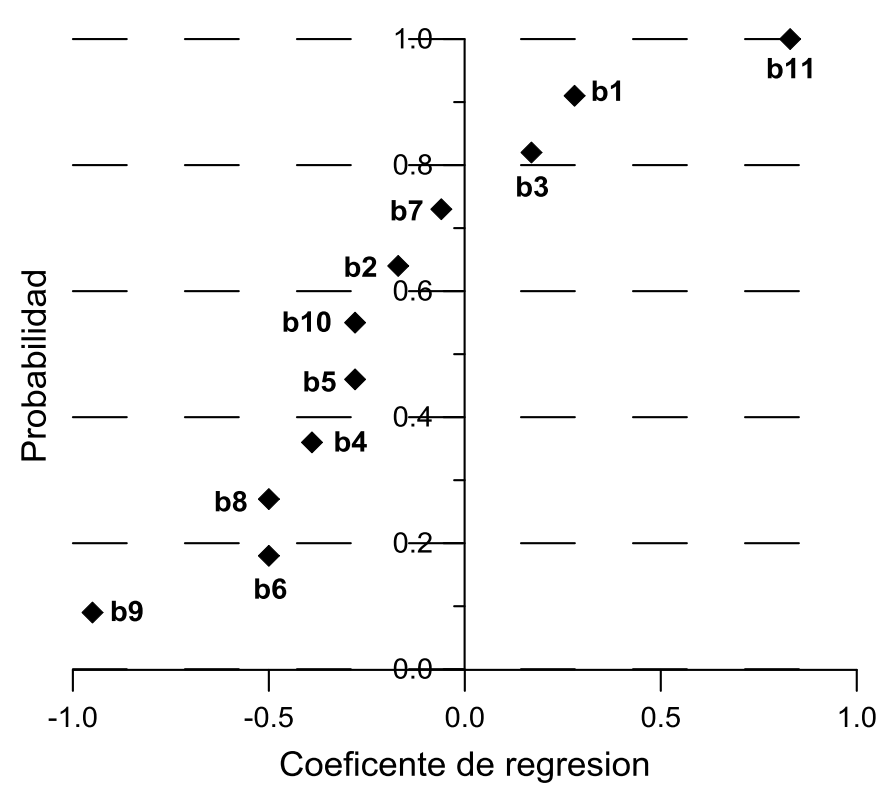

Figura 3. Pan de dulce: Normal probability plot de los coeficientes de regresión.

Para el pan de sal, los resultados obtenidos en la segunda etapa, diseño experimental por PlackettBurman por parámetro, de acuerdo a los valores corregidos en las gráficas de utilidad, fueron: en todos los experimentos el color y aroma obtuvieron su valor máximo, 1 ; en la textura las experimentos 2, 5, $69,10,11,12, R_{\mathrm{C} 1}$ y $R_{\mathrm{C} 2}$ alcanzaron el 1, el resto 0; en el sabor los experimentos $2,5,9,10, \mathrm{R}_{\mathrm{C} 1}$ y $\mathrm{R}_{\mathrm{C} 2}$ lograron 0,67 , los experimentos 3 y 11 alcanzaron 0,50 y el resto 0,33 ; en el costo los experimentos 2 , $3,5,6,7,8,9,10,12, \mathrm{R}_{\mathrm{C} 1}$ y $\mathrm{R}_{\mathrm{C} 2}$ obtuvieron 0,67 el experimento 11 tuvo 0,50 y el resto 0,33 y en el deseo de compra los experimentos $2,5,7, \mathrm{R}_{\mathrm{C} 1}$ y $\mathrm{R}_{\mathrm{C} 2}$ lograron el 1 el resto obtuvieron 0 . Los resultados totales por experimento fueron: experimento $1.0,44 ; 2$. 0,$89 ; 3$. 0,$53 ; 4$. 0,$44 ; 5.0,89 ; 6$. 0,67; 7. 0,67; 8. 0,$50 ; 9.0,89 ; 10.0,72 ; 11.0,67 ; 12.0,67 ; 13$. o $\mathrm{R}_{\mathrm{C} 1}$ (réplica al centro 1 ) 0,89 y 14 . o $\mathrm{R}_{\mathrm{C} 2}$ (réplica al centro 2) 0,89. Como se obtuvo el mismo resultado en las réplicas al centro, la desviación estándar y el error medido fueron de cero. Se observa las mayores puntuaciones en los productos donde los ingredientes estaban incorporados a un nivel medio.

De la matriz de diseño y el vector resultados se obtuvo el vector respuesta, integrado por los siguientes coeficientes por ingrediente variable: b1. harina de maíz $(0,14)$, b2. harina de plátano ($0,25)$, b3 . harina de cebada $(-0,47)$, b4 harina de soya $(0.31)$, b5 semillas de girasol $(0,14)$, b6 . ajonjolí $(-0,20)$, b7 pepa de zambo $(0,14)$, b8. brotes de alfalfa $(0,42)$, b9. quinoa $(0,58)$, b10 maní $(-$ $1,47)$ y b11 sal $(0,64)$. Es decir, los ingredientes de influencia positiva fueron: La harina de maíz (b1), la harina de soya (b4), las semillas de girasol (b5), la pepa de zambo (b7), los brotes de alfalfa (b8), la quinoa (b9) y la sal (b11). Los ingredientes con influencian negativa fueron la harina de plátano (b2), la harina de cebada (b3), el ajonjolí (b6) y el maní (b10). Como se puede observar en la gráfica normal probability plot (ver Fig. 4), de las 11 variables apenas una variable es significativa, el maní (b10). Este factor influye negativamente en el producto. 
MASKANA, Vol. 4, No. 1, 2013

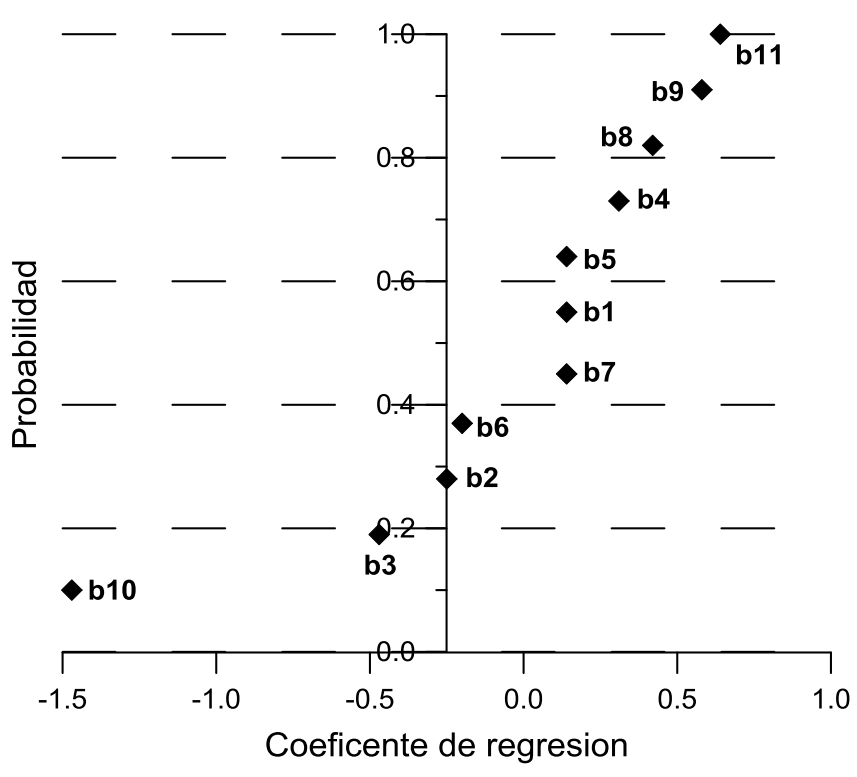

Figura 4. Pan de sal: Normal probability plot de los coeficientes de regresión.

\subsection{Optimización}

Para el pan de dulce, los resultados obtenidos en la segunda etapa, diseño experimental por PlackettBurman por parámetro, determinaron que como variables significantes al dulce de higo, negativo, y a las nueces, positivo. En esta ronda de 12 experimentos, se encontraron de acuerdo a los valores corregidos en las gráficas de utilidad: en todos los experimentos el color, textura y aroma obtuvieron su valor máximo, 1; en el sabor los experimentos $5,6,7, \mathrm{R}_{\mathrm{C} 1}, \mathrm{R}_{\mathrm{C} 2}, \mathrm{R}_{\mathrm{C} 3}$ y $\mathrm{R}_{\mathrm{C} 4}$ obtuvieron 1 y el resto de los experimentos 0,67 ; en el costo el experimento 5 obtuvo 1 , el experimento 8 el valor de 0,33 y el resto 0,67 y en el deseo de compra únicamente el experimento 8 obtuvo cero, el resto 1 . Los resultados obtenidos por experimentos fueron: 1 . 0,$89 ; 2$. 0,$89 ; 3$. 0,$89 ; 4$. 0,$89 ; 5.1,00 ; 6.0,95 ; 7$. 0,95; 8. 0,67; $\mathrm{R}_{\mathrm{C} 1} .0,95 ; \mathrm{R}_{\mathrm{C} 2} .0,95 ; \mathrm{R}_{\mathrm{C} 3} .0,95$ y $\mathrm{R}_{\mathrm{C} 4} .0,95$. Las cuatro réplicas al centro resultaron iguales luego la desviación y el error son cero. Los mayores resultados obtenidos estuvieron en las réplicas al centro y en el experimento 5 que incluyó $-\sqrt{2}$ de dulce de higos y las nueces al centro.

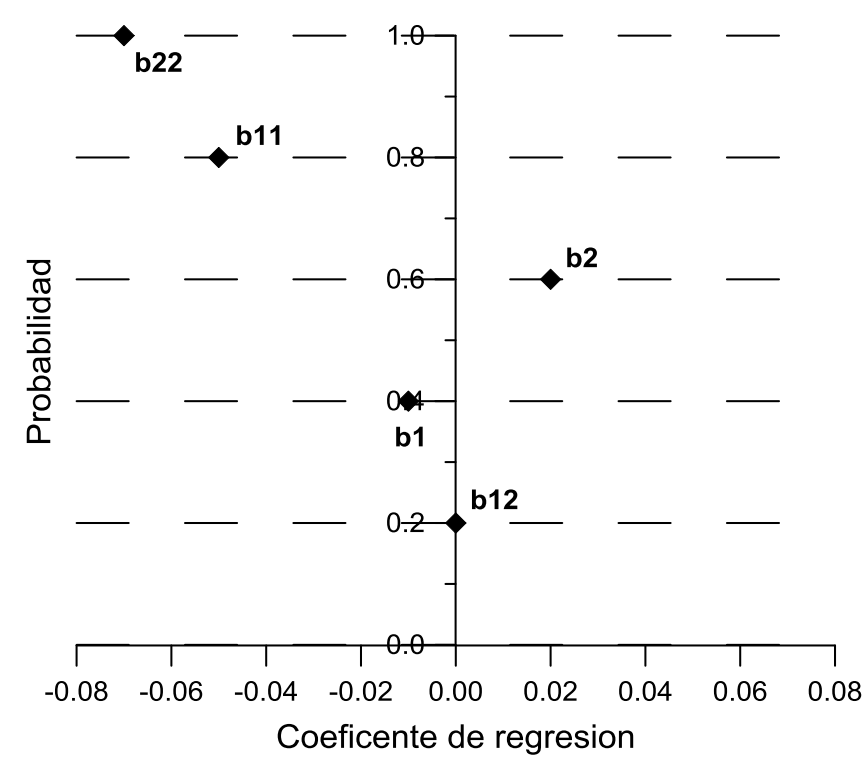

Figura 5. Pan de dulce, optimización: Normal probability plot de los coeficientes de regresión. 
De la gráfica de superficie de respuesta se pudo evidenciar que el pico máximo se alcanza en el punto medio de los higos (0), y en una cantidad ligeramente superior del centro (0) en las nueces. Por lo que finalmente solo las nueces son relevantes. Los coeficientes obtenidos de la matriz de superficie de respuesta y el vector resultado arrojaron los siguientes coeficientes: $\mathrm{b} 0=0,94 ; \mathrm{b} 1=-0,011 ; \mathrm{b} 1^{2}=-$ 0,$05 ; \mathrm{b} 12=0,00 ; \mathrm{b} 2=0,02$ y $\mathrm{b} 2^{2}=-0,07$; donde 1 representa al dulce de higo y 2 a las nueces (ver Fig. 5).

De la gráfica de superficie de respuesta y de la anterior concluimos que fueron relevantes: las nueces (b2) y las nueces al cuadrado (b2 ${ }^{2}$ ), mientras que el dulce de higo es irrelevante (b1). Luego, la ecuación resultante fue: $0,94+0,0151$ nueces $-0,068$ nueces $^{2}$. Al derivar ésta, para optimizar la función, se obtuvo $11 \mathrm{~g}$ de nueces, cantidad mayor al centro $8,8 \mathrm{~g}$. Todo el resto de ingredientes debe ser incorporado en sus puntos medios o al centro. La dosificación resultante optimizada fue: harina de trigo $50 \mathrm{~g}$; harina de maíz $14,25 \mathrm{~g}$; harina de plátano $9 \mathrm{~g}$; harina de cebada $2,25 \mathrm{~g}$; harina de soya 1,5 g; almidón de achira $10,5 \mathrm{~g}$; levadura $4 \mathrm{~g}$; aceite de soya $6 \mathrm{~g}$; huevos $10 \mathrm{~g}$; leche $20 \mathrm{~g}$; agua $25 \mathrm{~g}$; sal $0,4 \mathrm{~g}$; azúcar morena $18,75 \mathrm{~g}$; zumo de naranja $15 \mathrm{~g}$; pasas 7,5 g; higo en panela troceado $15 \mathrm{~g}$; manzanas $15 \mathrm{~g}$; y nueces $8,8 \mathrm{~g}$.

Para el pan de sal, los resultados obtenidos en la segunda etapa, determinaron como variable significante al maní, el cual tiene una influencia negativa. En esta ronda de 5 experimentos, se encontraron de acuerdo a los valores corregidos en las gráficas de utilidad: color, textura, aroma y deseo de compra 1 en todos los experimentos; sabor 4 y 5 obtuvieron 0,67 y el resto 1 y en el costo 1 y 2 alcanzaron 1 el resto 0.67 . Estos resultados determinaron los puntajes totales para los experimentos: 1. $1 ; 2.1 ; 3$. 0,$95 ; 4$. 0,89 y 50,89 . Las réplicas obtuvieron el mismo valor por tanto la desviación y el error fueron cero. Como se observa las mayores puntaciones correspondieron a las formulaciones con menos cantidad de maní; esto es -3 y -2. Para encontrar la ecuación óptima de maní en la fórmula se graficó de forma polinomial los resultados versus la cantidad de maní $[-3,-2,-1,0,0]$.

Para optimizar el pan de sal, derivamos la ecuación obtenida de la regresión polinomial que fue $=$ - 0,0275maní - 0,1395maní - 0,8295; donde el óptimo del maní resultó -2,54, cantidad equivalente a $0,35 \mathrm{~g}$. Por lo tanto, el resto de ingredientes deben ser incorporados en sus puntos medios o al centro. La dosificación resultante optimizada fue: harina de trigo $50 \mathrm{~g}$; harina de maíz 22,5 g; harina de plátano 7,5 g; harina de cebada 2,25 g; harina de soya $3,75 \mathrm{~g}$; levadura $3 \mathrm{~g}$; aceite de soya $6 \mathrm{~g}$; huevos $10 \mathrm{~g}$; leche $20 \mathrm{~g}$; agua $35 \mathrm{~g}$; sal 0,8 g; azúcar morena $5 \mathrm{~g}$; semillas de girasol 3,75 g; ajonjolí $3,75 \mathrm{~g}$; pepa de zambo $3,75 \mathrm{~g}$; brotes de alfalfa $2,25 \mathrm{~g}$; quinoa $3,75 \mathrm{~g}$ y maní $0,35 \mathrm{~g}$.

\subsection{Pruebas de aceptación}

Para verificar el grado de aceptación, se realizaron nuevas encuestas sobre los productos realizados con la fórmula optimizada. Los resultados obtenidos tanto en el pan de dulce como en el pan de sal fueron puntuaciones de 1 en todos los parámetros: color, textura, sabor, aroma, costo y deseo de compra; con lo que se supera el objetivo de obtener resultados superiores a 0,97. Cumpliéndose la aspiración de dosificar una fórmula que tuviera buena aceptación sensorial así como un precio atractivo a los posibles compradores.

El pan de dulce fue catalogado de la siguiente manera: El 50\% de encuestados afirmó que el color es excelente, en tanto que un 51,7\% les pareció buena la textura, un 63,3\% encontró excelente el sabor y un $45 \%$ bueno el olor, en cuanto al costo fue aceptado por el $55 \%$ como excelente. Por otra parte, un $90 \%$ afirma que compraría el producto, con una frecuencia semanal del $40 \%$ y eventual del $45 \%, 15 \%$ indicó una frecuencia diaria. Mientras que el pan de sal, un 60,3\% afirmó que el color es bueno, un $66,7 \%$ indicó como buena la textura, un $49 \%$ encontró bueno el sabor y un $57 \%$ bueno el olor. El costo fue aprobado como bueno por el 59\%. Un 78\% compraría el producto, el 34\% semanalmente, el 56\% eventualmente y el $10 \%$ diariamente. 


\section{CONCLUSIONES}

Aplicando el diseño experimental, se optimizaron las dos formulaciones planteadas inicialmente, cada una con 11 ingredientes variables, con un mínimo de recursos. En el pan de dulce, los ingredientes que influyen positivamente fueron: la harina de maíz, la harina de cebada y sobre todo las nueces, mientras los ingredientes que los hacían negativamente resultaron: la harina de plátano, la harina de soya, el almidón de achira, el azúcar morena, el zumo de naranja, las pasas, los higos en panela y las manzanas en canela. En cuanto al pan de sal, los ingredientes que influyen positivamente fueron la harina de maíz, la harina de soya, las semillas de girasol, la pepa de zambo, los brotes de alfalfa y la quínoa, mientras los ingredientes con influencian negativa resultaron: la harina de plátano, la harina de cebada, el ajonjolí y el maní. En ambos panes, luego de la experimentación se encontró un solo ingrediente influyente. Esto es, nueces para el pan de dulce, de influencia positiva y maní en el pan de sal de influencia negativa. El pan de dulce recibió una aceptación del 90\%, y el sabor fue aprobado por un $91 \%$ de los catadores. El pan de sal fue aprobado por un 78\%, el sabor fue considerado excelente o bueno por un $75,5 \%$. En ambos lo que menor aprobación recibió fue la textura.

Se comprobó que el diseño experimental es una alternativa adecuada para realizar dosificaciones de nuevos productos en la tecnología de alimentos, pues permite obtener resultados óptimos con un mínimo de recursos. Las variables involucradas pueden ser desde ingredientes hasta parámetros para el proceso que se quieren optimizar.

\section{BIBLIOGRAFÍA}

Badui, S., 1999. Química de los Alimentos (3ª ed.). Pearson Educación, Editorial Alhambra, México, págs. 409-411.

Bourdieu, P., 1998. La distinción, criterios y bases sociales del gusto. Taurus Ediciones, Madrid, 583 págs.

Box, G., S. Hunter, W. Hunter, 2008. Estadística para investigadores: Diseño, innovación y descubrimiento ( $2^{\mathrm{a}}$ ed.). Editorial Reverté, Barcelona, 639 págs.

Castaño, E., J. Domínguez, 2010. Diseño de experimentos: Estrategias y análisis en Ciencia y Tecnología. Universidad Autónoma de Querétaro, Querétaro, 428 págs.

Consonni, V., R. Todeschini, 1998. Pianificazione degli esperimenti. Milano Chemometrics and QSAR Research Group, Dipartimento di Scienze dell'Ambiente e del Territorio e di Scienze della Terra, Università Milano-Bicocca, Milano, 123 págs.

Darghan, E., S. Sinha, A. Goitia, 1978. Score test para el efecto del coeficiente de solapamiento en modelos de superficies de respuesta de primer y segundo orden. Dyna, 165, 234-245.

Garrido, F., S. González, M. Dondero, E. Wittig de Penna, 2010. Pasta untable de pavo enriquecida con fibras, vitaminas y minerales antioxidantes como ingredientes funcionales para el adulto mayor. Rev. Chil. Nutr., 37(3), 360-368.

Güemes, N., A. Totosaus, J. Hernández, S. Soto, E. Aquino, 2009. Propiedades de textura de masa y pan dulce tipo "Concha" fortificados con proteínas de suero de leche. Ciência e Tecnologia de Alimentos, 29(1), 70-75.

Lewis, G., D. Mathieu, R. Phan-Tan-Luu, 1999. Pharmaceutical experimental design. Marcel Dekker Inc., New York, 498 págs.

Marengo, E., 1994. Ottimizzaaione di processo e pianificazione sperimentale: Optimization and complex system analysis by chemometryc methods. Talete S.r.l., Milano.

Matos, A., K. Muñoz, 2010. Elaboración de pan con sustitución parcial de harina pre cocida de ñuña (Phaseoleusvulgaris L.) y tarwi (Lupinus mutabilis). Ciencia y Tecnología de Alimentos, 1(1), 3135 . 
Mesas, J., M. Alegre, 2002. El pan y su proceso de elaboración. Ciencia y Tecnología Alimentaria, 3(5), 307-313.

Navidi, W.C., 2006. Estadística para Ingenieros y Científicos. McGraw-Hill Interamericana de España S.L., 868 págs.

Pedhazur, E., L. Pedhazur, 1991. Measurement, design, and analysis: An integrated approach. Lawrence Erlbaum Associates, Incorporated, New York, 819 págs.

Pérez, C., 2006. Técnicas de análisis multivariante de datos: Aplicaciones con SPSS. Universidad de Complutense de Madrid ( $1^{\text {a }}$ ed.), Pearson Prentice Hall, Madrid, págs. 595-603.

Quaglia, G., 1991. Ciencia y tecnología de la panificación ( $2^{\mathrm{a}}$ ed.), traducido por Mateos-Nevado, B., Edición Acribia S.A., Zaragoza, España, 485 págs.

Rojas, C., L. Aguilar, P. Tripaldi, 2010. Desarrollo y optimización de un producto untable a base de soya. Revista Tecnológica ESPOL, 23(2), 33-40.

Rojas, C., P. Tripaldi, H. Dután, 2010. Desarrollo y optimización de una infusión aromática tipo tisana aplicando diseño de Plackett-Burman y optimización de máxima pendiente. Revista de Ciencias, $14,103-115$.

Rojas, C., P. Tripaldi, A. Pérez, P. Quinteros, 2012. Diseño experimental y métodos de decisión multicriterio para optimizar la composición de helado mantecado. Scientia Agropecuaria, 51-60.

Salamanca, G., M. Osorio, L. Montoya, 2010. Elaboración de una bebida funcional de alto valor biológico a base de borojó (Borjoa Patinoi Cuatrec). Rev. Chil. Nutr., 37(1), 87-96.

Saltos, H., A. Bayas, 2010. Aplicación de un diseño experimental de mezclas en el desarrollo de una "Barra Energética" con base en el salvado de palmito de pejibaye (Bactris gasipaes H.B.K). Revista Tecnológica ESPOL, 23(2), 1-8.

Sanchez, H., C. Osella, M. De la Torre, 2002. Optimization of gluten-free bread prepared from cornstarch, rice flour, and cassava starch. J. Food Sci., 67(1), 416-419.

Sancho, J., E. Bota, J. Castro, 2002. Introducción al análisis sensorial de los alimentos. Acervo General - ITESO, 336 págs.

Tejero, F., 1995. Panadería Española (2 Tomos). Editorial Montagud, Barcelona, 190 págs.

Tinoco, M., C. Rojas, P. Tripaldi, M. Criollo, L. Huayasaca, 2011. Aplicación de funciones de decisión multicriterio y diseño Plackett-Burman para el estudio de la calidad sensorial de mortadelas. Revista Venezolana de Ciencia y Tecnología de Alimentos, 1(2), 142-157.

Todeschini, R., 1998a. Definizioni ed analisi delle caratteristiche olfattive e gustative degli alimenti. Chitiote Editori, Pinerolo, 352 págs.

Todeschini, R., 1998b. Le metodologie della ricerca sperimentale: Esercizi. En: Todeschini, R. Introduzione alla chemiometria. EdiSES, Napoli, págs. 54-70.

Vaclavik, V., 2002. Fundamentos de la ciencia de los alimentos. Editorial Acribia S.A., Zaragoza, 146 págs. 